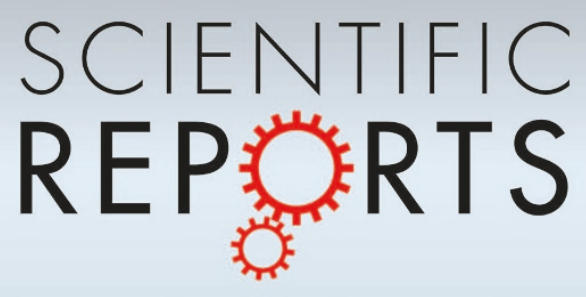

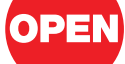

SUBJECT AREAS:

NEUROPSYCHIATRIC

DISORDERS

COGNITIVE NEUROSCIENCE

EMOTION

PSYCHOLOGY

Received

27 September 2012

Accepted

13 November 2012

Published

7 January 2013

Correspondence and requests for materials should be addressed to

E.L. (erwin.lemche@ kcl.ac.uk)

\title{
Somatization Severity Associated with Postero-Medial Complex Structures
}

\author{
Erwin Lemche ${ }^{1,4}$, Vincent P. Giampietro ${ }^{2}$, Michael J. Brammer ${ }^{2}$, Simon A. Surguladze ${ }^{2}$, \\ Steven C. R. Williams ${ }^{2} \&$ Mary L. Phillips ${ }^{3}$
}

\begin{abstract}
'Section of Cognitive Neuropsychiatry, Institute of Psychiatry, King's College Medical School, London SE5 8AF, United Kingdom, ${ }^{2}$ Department of Neuroimaging Sciences, Institute of Psychiatry, King's College Medical School, London SE5 8AF, United Kingdom, ${ }^{3}$ Western Psychiatric Institute and Clinic, University of Pittsburgh School of Medicine, Pittsburgh PA 15213, United States of America, ${ }^{4}$ Faculty of Psychology, The University of Constance, 78457 Konstanz, Germany.
\end{abstract}

Somatisation is a frequent problem in various psychiatric disorders, yet the cerebral mechanisms of somatisation remain unexamined. To test if somatisation is susceptible to emotional states, we investigated relationships between somatisation severity, neural effective connectivity, and autonomic responses to emotional facial expressions. Volunteering participants $(\mathrm{N}=20)$ were presented with facial expressions of happy and sad emotion at three intensity levels (0\%-50\%-100\%) in a fast implicit ER-fMRI design with concurrent derivation of skin conductance levels (SCL). Self-reported somatisation severity as assessed with Riefs SOMS-2 index was correlated with neural response controlling for other clinical traits to ascertain brain bases of somatisation. Regression analyses estimated effective connectivity of main clusters so determined with peripheral autonomic responses. Regions in which magnitude of activity correlated with somatisation severity consisted in both happy and sad conditions of the anterior ventral precuneus (BA7), along with posterior cingulate gyrus (PCC, BA23, sad condition) and anteromedial thalamus (happy condition).

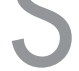

omatisation in the modern sense came into official life with DSM-III-R as a subset of somatoform disorders, in the consensus that medically unexplained symptoms constitute its key observation criterion ${ }^{1}$. Hence, somatisation disorder was originally defined as a condition with "multiple physical symptoms but no apparent physical disease"2. These health complaints typically refer to gastrointestinal, uro-genital, cardio-respiratory, other internal systems, or musculoskeletal problems ${ }^{1}$. Subsequently, it was shown that such unexplained symptoms are typically paired with psychological distress, namely anxiety and/or depression, and frequently also with axis II diagnoses ${ }^{3-5}$. The more general category "somatoform disorders", in contrast, comprises a very heterogeneous group of patient presentations, ranging from motor conversion disorders to hypochondriasis, to somatisation disorder, to body dysmorphic, and to pain disorders ${ }^{6}$. The current definition of somatisation is the tendency to experience and communicate problems in internal organ systems in response to emotional stress ${ }^{7}$. We refer in the following paragraphs to somatisation as the complaint about multiple somatic symptoms accompanied by psychological distress ${ }^{8}$.

Heightened autonomic (ANS) arousal and cognitive filtering of bodily sensations have been identified as underlying bodily dysregulation, pain, and medically unexplained physical symptoms in somatisation ${ }^{1}$. In several clinical groups, somatisation of chronic pain tendencies coincided with hypercortisolism in the hypothalamic pituitary adrenocortical axis (HPA $)^{9}$, which can aggravate respective illnesses with an inflammatory component $^{6,10}$. Genetic association studies have linked the somatisation trait (as measured with Riefs Somatization Index SOMS-2 or the somatisation subscale of Derogatis' SCL-90) significantly to the long alleles of the serotonin transporter (5-HTTLPR, SLC6A4, chromosome 17) (11,12 $^{2}$ and serotonin receptor type 2A (HTR2A, chromosome 13) ${ }^{12,13}$ genes. Furthermore, individuals with somatisation syndrome showed different immunological alterations ${ }^{14}$, and greater tryptophan depletion effects ${ }^{6,15,16}$, relative to individuals with major depression. Tryptophan, serotonin, and branched-chain monoamine levels have also been found to be decreased in somatisation disorders ${ }^{15}$, and thus low levels of serotoninergic amino acids have been considered to be correlates of somatisation ${ }^{13,16}$.

A recent review on neuroimaging in somatoform disorders ${ }^{17}$ lists only three neuroimaging studies on somatisation disorder and concludes that the state of research in this disorder is inconsistent and unsatisfactory. Another previous neuroimaging study has, in parallel, shown that somatisation accompanied by anxiety is associated with activity in interoceptive regions, including ventrolateral prefrontal, subgenual anterior cingulate 


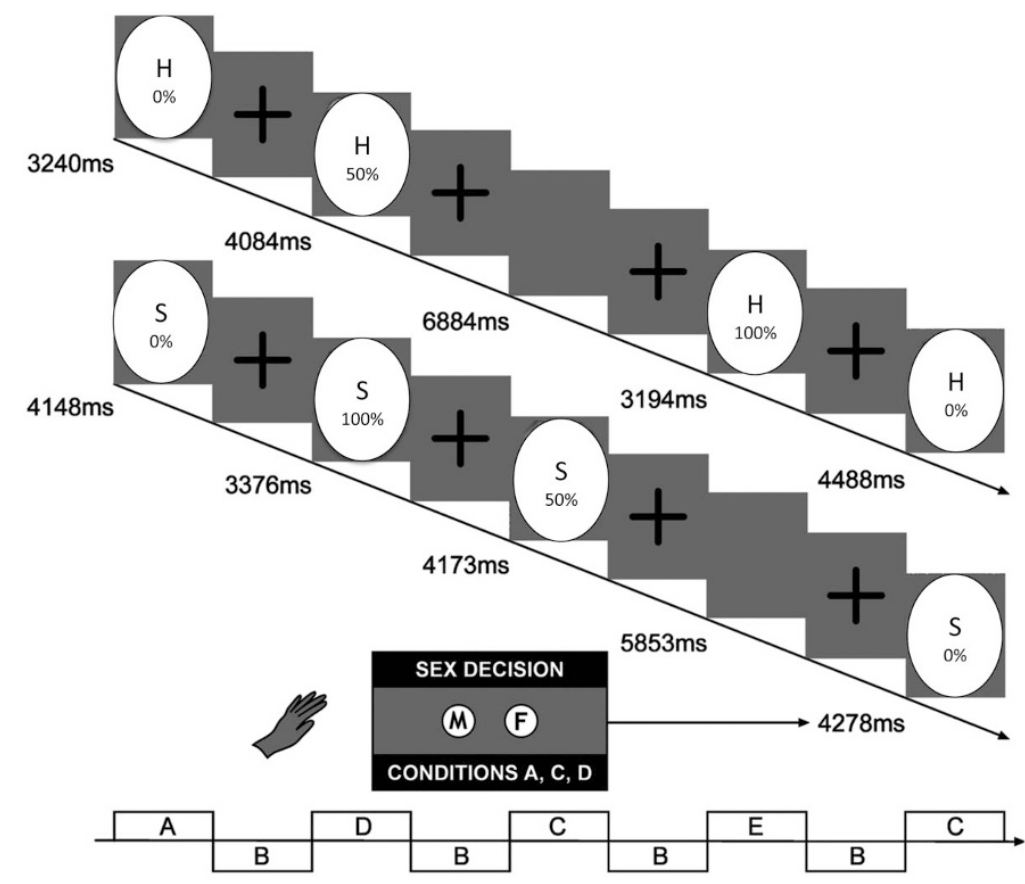

Figure $1 \mid$ Experimental design diagram for the implicit facial expression task. Intensity-morphed facial expressions were presented to the participants in two separate runs, one with happy, and one with sad faces ${ }^{25}$. Following instructions, the subjects had to determine the sex of the face, rather than to judge the emotion (implicit task).

gyral, as well as anterior insular cerebral metabolic activity ${ }^{18}$. These regions are innervated by serotoninergic axons ascending from the rostral dorsal raphe nucleus system.

In the present study, we examined whether somatisation is associated with abnormal activity in regions supporting interoception, and heightened autonomic responses, to external displays of emotion frequently conveyed in social encounters-facial expressions. We used a definition of somatisation according to established DSM-IV and ICD-10 criteria, the Screening for Somatoform Disorders $\left(\right.$ SOMS-2) ${ }^{19}$. The derived Complaint Somatization Index reflects the sum of the physical symptoms without medical explanation, and therefore describes a general tendency towards somatisation in individuals, according to its authors ${ }^{20}$. Because somatisation is a phenomenon known to appear in several psychopathological categories (e.g. anxiety, depression, somatoform disorders, schizophrenia, dementias etc.), we applied a cross-sectional observational design to a group of non-clinical and pre-clinical volunteers, in the first instance.

We presented participants with facial expressions of emotions of opposing valence (happy and sad), and examined respective relationships between associated neural activity, self-reported somatisation, and simultaneously-measured relative skin conductance level (rSCL), as a measure of individual sympathetic arousal. We relied on a paradigm with well-known neural response properties (e.g. limbic activation in anxiety or depression), and with typically overall sparse activation patterns. Facial expressions were chosen to induce socially conveyed emotional activation. This rationale followed the clinical insight reported in the literature, namely the point that somatisation may be a reaction style to social-emotional stressors ${ }^{7}$.

Existing findings led us to assume that greater level of somatisation trait would be associated with elevated activity in serotonergicallymodulated neural regions subserving interoception of bodily signals, and elevated autonomic responses, to these external displays of emotion. Specifically, we hypothesized (i) that the degree of somatisation severity would co-vary with cerebral activation in regions known to be involved in interoception, e.g. anterior/posterior cingulate, and anterior insular regions. Furthermore, as suggested by replicated findings on alterations in autonomic arousal involved in somatisation, we hypothesized (ii) a role of the ANS in regulating somatisation severity.

Table 1 | Main correlation regions of emotion and somatisation severity

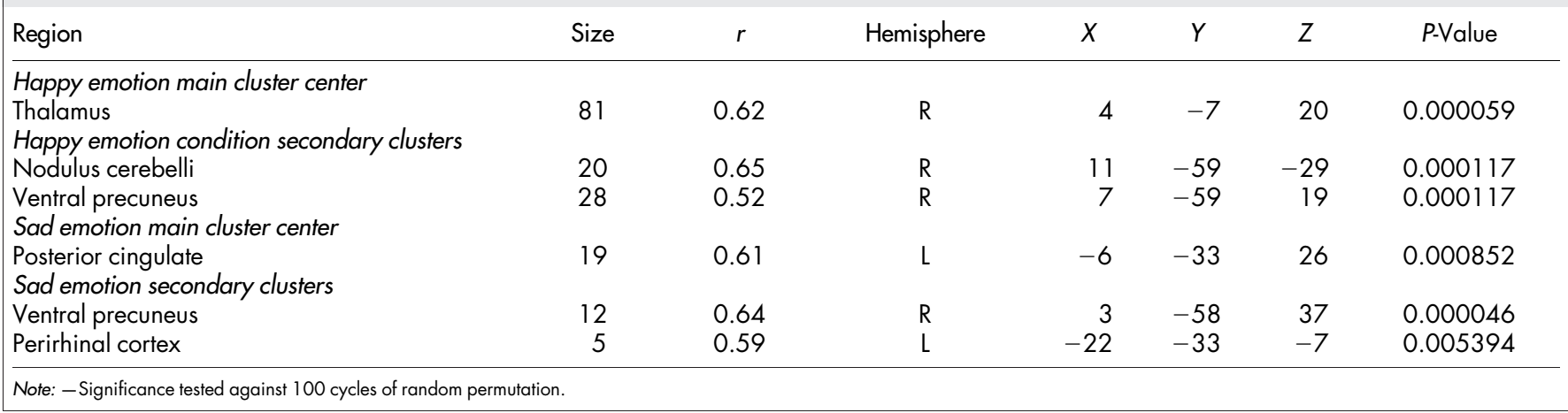




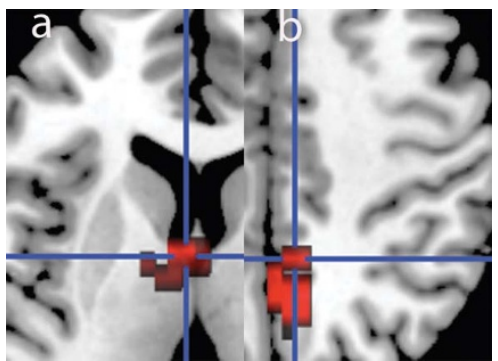

Figure 2 | Main correlation regions of emotion and somatisation severity. Visualization of main clusters in happy (Panel a, left) and sad facial expression stimulation condition (Panel b, right). Happy: cluster center in the right anteromedial thalamus (axial view). Sad: cluster center in left PCC (BA23), extending into precuneus (BA7). For details see Table 1 (radiological convention, $\mathrm{L}=\mathrm{R}$ ).

\section{Results}

Self-reported severity of somatisation. Overall, somatisation severity in this group was low through severe $(5.14 \pm 7.78, \mathrm{~m} \pm$ s.d., score points, range $0-31$ ), and six participants fulfilled criteria for chronic somatizers' status. Internal consistency of the somatisation scale (Cronbach's $\alpha=0.783$ ) was sufficient for further analyses. Age, but none of the other sociodemographic variables, was significantly correlated with somatisation severity $(r=0.469, P=0.018$, two tailed). Sex effects could not be detected in any self-report scale, nor in any of the neural or autonomic signal variables. Because of the intercorrelation of the clinical scales mentioned (below in the Methods section) with self-reported somatisation severity ( $P_{\mathrm{s}}<$ 0.05 ), the variances of these instruments (raw scores) and of subjects' age were removed in partial correlation images (Table 1).

Behavioural and psychophysiological data. The judgment accuracy $(50.59 \%)$ for the gender recognition was around the chance level of $50 \%$, and well in line with studies using similar fast presentation paradigms. Also, the response times were in good accordance with previous investigations. The average $( \pm$ s.d.) values for rSCL in MSiemens: $100 \%$ Happiness 3.019 ( \pm 0.0044$) ; 50 \%$ happiness 3.010 $( \pm 0.0476), 0 \%$ happiness $2.996( \pm 0.0416) ; 100 \%$ sadness 3.012 $( \pm 0.0107) ; 50 \%$ sadness $3.008( \pm 0.0054) ; 0 \%$ sadness $2.995( \pm 0.0364)$.

Functional connectivity between main clusters. BOLD signal levels extracted from the main clusters in each emotion category were used to test for functional connectivity between these clusters in the respective condition. To the happy emotion condition, there were three distinct clusters of activity: right anteromedial thalamic cluster (Talairach coordinates $X Y Z, 47$ 20, Figure 2a); left anterior ventral precuneus (Brodmann Area BA7; $7-59$ 19); and right cerebellar nodulus $(11-59-29)$. These three clusters showed functional connectivity, as indicated by association of their BOLD signal levels: anterior thalamus correlated with anterior ventral precuneus ( $r=0.776, P<0.0001$, two-tailed) and cerebellar nodulus $(r=0.422$, $P<0.033$, two-tailed). Right cerebellar nodulus correlated with precuneus ( $r=0.679, P<0.0001$, two-tailed).

To the sad emotion condition, there were the following clusters of activity (Figure 2b): A left posterior cingulate gyral cluster (PCC, BA23; $-6-3326$ ), contingent dorsally with a cluster in the right anterior ventral portion of the precuneus (BA7, 3 -58 37); and a cluster in the left mesial-to-caudal surface of the perirhinal cortex (BA35, -22 - 33 -7) situated ventrally to the PCC cluster. Testing for functional connectivity amongst the three clusters revealed that PCC had functional connectivity with perirhinal cortex $(r=0.677$, $P<0.001$, two-tailed); and in turn, the perirhinal area had functional connectivity with the ventral precuneus $(r=0.383, P<0.048$, twotailed). The properties of the correlation image clusters are summarized in Table 1.
Effective connectivity between neural, autonomic and behavioural measures. Linear hierarchical regression analyses were used to estimate causal associations between neural activation in the main correlation clusters, physiological and behavioural measures, because the regression method can incorporate behavioural measurements. In each case, a mediatory relationship was observed between (a) neural response from the main clusters predicting rSCL; (b) neural response in the main clusters predicting somatisation severity; and (c) rSCL predicting somatisation severity: Thalamus signal predicted somatisation severity (adjusted $R^{2}=0.518, F=5.834, P<0.006$ ) in the happy condition. Happiness $100 \%$ relative skin conductance level (rSCL) predicted somatisation severity (adjusted $R^{2}=0.520, F=5.330$, $P<0.011$ ), as did 50\% rSCL (adjusted $R^{2}=0.940, F=12.387, P<0.001$ ). Thalamus signal predicted $100 \%$ (adjusted $R^{2}=0.782, F=29.716$, $P<0.0001$ ) and 50\% (adjusted $R^{2}=0.184, F=5.069, P<0.04$ ) rSCL in the happy condition. PCC signal predicted somatisation severity (adjusted $R^{2}=0.448, F=3.568, P<0.026$ ) in the sad condition. Sadness $100 \%$ rSCL predicted somatisation severity (adjusted $R^{2}=0.425$, $F=3.069, P<0.069$ ), as did 50\% rSCL (adjusted $R^{2}=0.535, F=6.136$, $P<0.006$ ). PCC signal predicted $100 \%$ (adjusted $R^{2}=0.415, F=4.370$, $P<0.015$ ) and $50 \%$ (adjusted $R^{2}=0.313, F=9.653, P<0.006$ ) rSCL in the sad condition ( $N=20$ for all reported models).

\section{Discussion}

We aimed to examine the neural mechanisms underlying the phenomenon of somatisation as measured with an internationally accepted and well-validated questionnaire. In both happy and sad emotion conditions, anterior ventral precuneus, and PCC (sad condition) and anteromedial thalamus (happy condition), were main clusters of activity. Anatomical connectivity had previously been reported amongst all regions here described. Our findings are consistent with the notion that somatisation may be characterized by mental rumination, implicating regions associated with autobiographical and self-referential memory, but similar across emotional valences.

We can therefore state that our hypothesis (i) was supported in part, by the involvement of the posterior cingulate region, but not so with respect to insular involvement. Hypothesis (ii) was supported by the finding that ANS outflow exhibited linear relationships with somatisation self-report at each level of emotional stimulation. This finding suggests a distinct role of the ANS in co-regulating somatisation severity. The negative finding of the insula as a main correlation region may perhaps appear as a consequence of our non-clinical sample. Insular activation would have appeared only if we would have applied a more lenient threshold at $P<0.05$, but then with an expectable false-positive cluster rate of 5 , and only in the sad condition. Such a finding would therefore not be conservative enough to reject type I error with certainty. In this analysis, however, we have applied the strictest possible statistical thresholds, and there were no false positives expectable by chance. At this level of stringency, ventral precuneus was the common denominator across emotion category.

The precuneus has been identified as one of four "high centrality hub regions" using diffusion tensor imaging network analyses ${ }^{28}$. The precuneus is also considered a cerebral 'hot spot' in terms of heightened metabolic rate, where deactivation corresponds to loss of consciousness under anesthesia. Resting state analyses have further divided the precuneus into subsections. In its anterior portion it mediates mainly self-referential functioning, whereas the posterior portion is involved in autobiographic memory ${ }^{29}$. The anterior portion is also assumed to be involved in sensorimotor control. The ventral portion (as observed here) is strongly interconnected with the PCC (consistent with our present findings), and is assumed to be part of the limbic system circuit ${ }^{30}$. The ventral sector corresponds in combination with PCC to the postero-medial complex (PMC) in primates, which has been replicated to exist in humans ${ }^{30}$. The 
PMC is altered in psychiatric disorders such as attention-deficit hyperactivity disorder, early onset Alzheimer's disease, and diabetic encephalopathy. The PCC is an evaluative interoceptive reasoning region that receives input from the anterior thalamic nuclei, which are conceived to subserve emotion and memory functions. We consider our findings related to recent default mode network findings concerning the PMC.

The perirhinal cortex is closely related to the parahippocampal gyrus and has an integrative role in hippocampal memory storage processes. It receives input from almost every higher-order cognitive region, and spatial orientation is disturbed, if the anatomical connection to the PCC is interrupted. It is possible that spatial orientation processes within face processing could play a role in our results. The cerebellum is implicated in control of cognitive and motor systems. Parahippocampal structures and cerebellum share genetic influences with the precuneus/posterior cingulate system ${ }^{31}$, besides anatomical connectivity. All structures are part of either the rostral (thalamus, PCC, entorhinal area, precuneus) or caudal (cerebellum) branches of the dorsal raphe system. Also, the precuneus is a serotoninergic structure, as serotonin transporter binding has been demonstrated in the parietal cortex including the precuneus ${ }^{32}$.

This study is limited by the fact that concurrent measurement of serotonin metabolism, transport or binding could not be performed. Future studies may be able to replicate the present findings in the context of an imaging genetics investigation for monoamine gene polymorphisms. In addition, it remains to be investigated whether there is a true correlation with the key regions here identified with interleaved resting state fMRI activation. Together, our findings suggest that a network of serotonergically-modulated neural regions, of which many are key components of the posterior default mode network, may play an important role in mediating vulnerability to complaint somatisation.

\section{Methods}

Participants. Twenty unselected volunteering right-handed individuals ( $\mathrm{m} \pm$ s.d., age $31.6 \pm 7.34$ years; education level, $2.50 \pm 0.60 ; 2=$ junior college level, 7 females) participated in the study. The volunteers had been interview-screened to exclude histories of major psychiatric, neurological or substance-abuse problems. All participants gave written informed consent to the scientific use of their data, and were reimbursed for their participation. The Bethlem Royal and Maudsley Ethics Committee (Research) had endorsed all experimental procedures. Somatisation severity was assessed with Rief's Complaint Somatization Index ${ }^{19}$. We preferred an observational cross-sectional design over a case-control design. Instead we attempted to "purify" the somatisation measure as much as possible from detected co-variation with other clinical symptoms. Subjects therefore also completed the following selfreport questionnaires for statistical control purposes: Dissociative Experience Scale, Cambridge Depersonalization Scale, Beck Depression Inventory, Toronto Alexithymia Scale, Spielberger State-Trait Anxiety Inventory, among others. Co-variances of these were then removed in analyses.

\section{Description of the SOMS-2 instrument. The instrument "Screening for} Somatoform Disorders" (SOMS-2) ${ }^{19}$ is a self-report questionnaire that allows objectifying abnormal somatisation tendencies in patients. As mentioned above, SOMS-2 contains a comprehensive list of 53 physical symptoms based on DSM-IV and ICD-10 diagnostic criteria in the past two years, for which no organic cause was found. In this self-report, individuals state whether a symptom was present or not (Guttman-type response format). Furthermore, the SOMS-2 contains statements referring to the other somatoform disorders (hypochondriasis, body dysmorphic disorder, pain disorders). The instrument evaluates subjective complaints, the degree of impairments caused by the reported physical symptoms, and possible differential diagnostic restrictions. Its reliability and validity has been established in more than 300 Patients $^{21}$. The resulting measure derived from number, severity, and duration of somatic symptoms is called Complaint Somatization Index. It conforms to the ICD10 criteria of somatisation disorder. As a pathological cutoff, a score $>17$ points was used, in accordance with the SOMS-2 manual. Healthy controls have an average of 6.1 , whereas psychiatric inpatients have a mean of 20.2. In a representative study of 2050 persons $^{22}, 484$ or $23.6 \%$ fulfilled this criterion for the status of chronic somatisation. This rate is approximately consistent with the proportion of chronic somatizers in our sample.

Experiments. Happy and sad facial expressions of emotion in $0 \%-50 \%-100 \%$ gradations of intensity were presented in two separate implicit ER-fMRI tasks. Each 6-minute experiment comprised 10 facial identities at each intensity level that were visually presented for $2 \mathrm{~s}$ with random inter-stimulus intervals (ISI) averaging $\lambda=4.9 \mathrm{~s}$. The ISIs comprised a fixation cross to maintain subjects' focus of attention (60 facial stimuli, 12 non-facial stimuli), as previously described ${ }^{23,24}$. A uniform computer-morphed version of Ekman's affective faces was employed ${ }^{25}$ with all nonfacial components removed. A design diagram of the paradigm is depicted in Figure 1. Under the assumption that the subtraction map reflects pure emotion-induced cerebral activation at higher intensity levels, $100 \%-50 \%$ aggregate images were computed, after removing neutral facial expression activation. These aggregate images, as previously described ${ }^{26}$, were used as the basis for correlation analysis with SOMS-2 scales.

Neuroimaging. $\mathrm{T}_{2}{ }^{*}$ and $\mathrm{T}_{2}$ high-resolution datasets (for spatial normalization) were acquired on a Neurovascular GE Signa 1.5 T MRI system with $40 \mathrm{~m} / \mathrm{mT}$ high-speed gradients: $\mathrm{T}_{2}{ }^{*}$ - 16 slices, slice thickness $7 \mathrm{~mm}(+0.7 \mathrm{~mm}$ gap), in-plane resolution $3.44 \mathrm{~mm}, \mathrm{~T}_{E} 40 \mathrm{~ms}, \mathrm{~T}_{R} 2,000 \mathrm{~ms}, \mathrm{FA} \alpha 70^{\circ}, 64^{2}$ matrix, FOV $25 \mathrm{~cm} ; \mathrm{T}_{2}-43$ slices, slice thickness $3 \mathrm{~mm}$ ( $+0.3 \mathrm{~mm}$ gap), in-plane resolution $1.72 \mathrm{~mm}, \mathrm{~T}_{E} 73 \mathrm{~ms}, \mathrm{~T}_{R}$ $16,000 \mathrm{~ms}, \mathrm{FA} \alpha 90^{\circ}, 128^{2}$ matrix, FOV $25 \mathrm{~cm}$.

Psychophysiological recording. Derivations of electrodermal activity (EDA) related to the task were made online during neuroimaging data acquisition. The methodology of simultaneous fMRI and psychophysiology data acquisition, filtering, and analysis used here has been published previously ${ }^{27}$. Applying criteria of 0.01 $\mu$ Siemens, EDA was analyzed in an event-related manner for each of the three different emotion expression intensities in each subject using the software program SC-ANALYZE (Neuroimaging Research Group, Institute of Psychiatry, London, UK). Latency windows of 1.2-3.3 s post stimulus onset were evaluated to ensure that EDA was not contaminated by nonspecific SCR discharges (increasing skin conductance level, SCL), such as those to the following stimulus. Following standard procedures, the relative SCL (rSCL) variable was computed expressing individual spans between minima and maxima within each condition, with the relative mean normalized to these, using the formula previously described ${ }^{27}$.

fMR image analysis. The statistical inference software package XBAM version 4.0 (Brain Image Analysis Unit, IOP London; www.brainmap.it), which implements mathematical control for signal-to-noise ratio, was used to analyze fMRI data. XBAM combines nonparametric permutation based resampling methods with GLM statistics, wavelet signal denoising methods, control of false-positive voxels and clusters, and reports exact significances rather than results corrected for family-wise error rates.

Correlation images. All reported results are corrected for optimal rates of expected false-positives of $\leq 1$ error 3D clusters over the whole brain volume (Table 1). We computed the Pearson product-moment correlation coefficient $r$ between the somatisation severity measures and blood oxygen level dependent (BOLD) effect data at each voxel. We then computed the null distribution of correlation coefficients by first permuting 100 times the somatisation measures between the subjects and recomputing $r$ for each data shuffle, before combining all the $r$ values into one large null distribution. Cluster level maps were generated by combining adjacent voxels where $r$ was found to be significant into 3D clusters and testing the cluster masses (sum of the $r$ values over the $3 \mathrm{D}$ cluster) for significance against the masses of $3 \mathrm{D}$ clusters occurring by chance in the permuted data. BOLD signal level, in mean percentage effect size, was extracted from the peak voxel in the main clusters-individually ascertained for each single subject.

1. Katon, W. et al. Somatization: a spectrum of severity. Am J Psychiatry 148, 34-40 (1991).

2. Smith, G. R. Jr., Monson, R. A. \& Ray, D. C. Psychiatric consultation in somatization disorder. A randomized controlled study. N Engl J Med 314, 1407-1413 (1986).

3. Allen, L. A., Woolfolk, R. L., Escobar, J. I., Gara, M. A. \& Hamer, R. M. Cognitivebehavioral therapy for somatization disorder: a randomized controlled trial. Arch Intern Med 166, 1512-1518 (2006).

4. Lieb, R., Meinlschmidt, G. \& Araya, R. Epidemiology of the association between somatoform disorders and anxiety and depressive disorders: an update. Psychosom Med 69, 860-863 (2007).

5. Bornstein, R. F. \& Gold, S. H. Comorbidity of personality disorders and somatization disorder: A meta-analytic review. Journal of Psychopathology and Behavioral Assessment 30, 154-161 (2008).

6. Dimsdale, J. E. \& Dantzer, R. A biological substrate for somatoform disorders: importance of pathophysiology. Psychosom Med 69, 850-854 (2007).

7. Lipowski, Z. J. Somatization: the concept and its clinical application. Am J Psychiatry 145, 1358-1368 (1988)

8. Rief, W. \& Auer, C. Cortisol and somatization. Biol Psychol 53, 13-23 (2000).

9. Anderson, R. U., Orenberg, E. K., Morey, A., Chavez, N. \& Chan, C. A. Stress induced hypothalamus-pituitary-adrenal axis responses and disturbances in psychological profiles in men with chronic prostatitis/chronic pelvic pain syndrome. J Urol 182, 2319-2324 (2009).

10. Nater, U. M., Gaab, J., Rief, W. \& Ehlert, U. Recent trends in behavioral medicine. Curr Opin Psychiatry 19, 180-183 (2006).

11. Hennings, A., Zill, P. \& Rief, W. Serotonin transporter gene promoter polymorphism and somatoform symptoms. J Clin Psychiatry 70, 1536-1539 (2009). 
12. Koh, K. B., Choi, E. H., Lee, Y. J. \& Han, M. Serotonin-related gene pathways associated with undifferentiated somatoform disorder. Psychiatry Res 189, 246-250 (2011).

13. Klengel, T. et al. Somatization in major depression--clinical features and genetic associations. Acta Psychiatr Scand 124, 317-328 (2011).

14. Rief, W. et al. Immunological differences between patients with major depression and somatization syndrome. Psychiatry Res 105, 165-174 (2001).

15. Rief, W. et al. Psychobiological aspects of somatoform disorders: contributions of monoaminergic transmitter systems. Neuropsychobiology 49, 24-29 (2004).

16. Maes, M., Galecki, P., Verkerk, R. \& Rief, W. Somatization, but not depression, is characterized by disorders in the tryptophan catabolite (TRYCAT) pathway, indicating increased indoleamine 2,3-dioxygenase and lowered kynurenine aminotransferase activity. Neuro Endocrinol Lett 32, 264-273 (2011).

17. Browning, M., Fletcher, P. \& Sharpe, M. Can neuroimaging help us to understand and classify somatoform disorders? A systematic and critical review. Psychosom Med 73, 173-184 (2011).

18. Brody, A. L. et al. Brain metabolic changes associated with symptom factor improvement in major depressive disorder. Biol Psychiatry 50, 171-178 (2001).

19. Rief, W., Hiller, W. \& Heuser, J. SOMS: The screening for somatoform disorders. (Hans Huber Verlag, 1997).

20. Rief, W. et al. The classification of multiple somatoform symptoms. J Nerv Ment Dis 184, 680-687 (1996).

21. Rief, W. \& Hiller, W. A new approach to the assessment of the treatment effects of somatoform disorders. Psychosomatics 44, 492-498 (2003).

22. Rief, W., Hessel, A. \& Braehler, E. Somatization symptoms and hypochondriacal features in the general population. Psychosom Med 63, 595-602 (2001).

23. Surguladze, S. A. et al. A differential modulation of visual cortical activation by displays of danger, reward and distress. NeuroImage 19, 1317-1328 (2003)

24. Surguladze, S. et al. A differential pattern of neural response toward sad versus happy facial expressions in major depressive disorder. Biological Psychiatry 57, 201-209 (2005).

25. Young, A. W., Perrett, D. I., Calder, A. J. \& Ekman, P. Facial expressions of emotion: stimuli and test (FEEST). (Thames Valley Test Company, 2002).

26. Lemche, E. et al. Cerebral and autonomic responses to emotional facial expressions in depersonalisation disorder. Br J Psychiatry 193, 222-228 (2008).

27. Lemche, E. et al. Human attachment security is mediated by the amygdala: evidence from combined fMRI and psychophysiological measures. Human Brain Mapping 27, 623-635 (2005).
28. Bullmore, E. \& Sporns, O. Complex brain networks: graph theoretical analysis of structural and functional systems. Nat Rev Neurosci 10, 186-198 (2009).

29. Sajonz, B. et al. Delineating self-referential processing from episodic memory retrieval: common and dissociable networks. Neuroimage 50, 1606-1617 (2010).

30. Margulies, D. S. et al. Precuneus shares intrinsic functional architecture in humans and monkeys. Proc Natl Acad Sci U S A 106, 20069-20074 (2009).

31. Glahn, D. C. et al. Genetic control over the resting brain. Proc Natl Acad Sci US A 107, 1223-1228 (2010).

32. Nakamura, K. et al. Brain serotonin and dopamine transporter bindings in adults with high-functioning autism. Arch Gen Psychiatry 67, 59-68 (2010).

\section{Acknowledgments}

This research was funded by the Wellcome Trust. We thank David Gasston and Jeffrey Dalton for technical assistance. This investigator-initiated study was supported by a grant of the Wellcome Trust to Drs Phillips and Lemche. The sponsor had no influence on design and conduct of the study; collection, management, analysis, and interpretation of the data; and preparation, review, or approval of the manuscript. The corresponding author had full access to all of the data in the study and takes responsibility for the integrity of the data and the accuracy of the data analysis.

\section{Author contributions}

Conceived and designed the experiments: SAS MLP EL. Performed the experiments: EL SCRW. Wrote custom software scripts: MJB VGP. Analyzed the data: EL VGP. Wrote the paper: EL MLP SAS MJB VGP. Recruited participants: EL.

\section{Additional information}

Competing financial interests: The authors declare no competing financial interests License: This work is licensed under a Creative Commons Attribution-NonCommercial-NoDerivs 3.0 Unported License. To view a copy of this license, visit http://creativecommons.org/licenses/by-nc-nd/3.0/

How to cite this article: Lemche, E. et al. Somatization Severity Associated with Postero-Medial Complex Structures. Sci. Rep. 3, 1032; DOI:10.1038/srep01032 (2013). 